\title{
In Vitro Hypoxia Responsiveness Of Fdg And Faza Retention: Influence Of Shaking Versus Stagnant Conditions, Glass Versus Polystyrene Substrata And Cell Number Down-Scaling
}

Morten Busk ( $\square$ morten@oncology.dk )

Aarhus University Hospital https://orcid.org/0000-0001-5711-1017

Michael R Horsman

Aarhus Universitetshospital

Jens Overgaard

Aarhus Universitetshospital

Steen Jakobsen

Aarhus Universitetshospital

Original research

Keywords: hypoxia, tracer availability, diffusion, convection, cell substrata, in vitro conditions

Posted Date: March 11th, 2020

DOI: https://doi.org/10.21203/rs.3.rs-16661/v1

License: (c) (1) This work is licensed under a Creative Commons Attribution 4.0 International License.

Read Full License 


\section{Abstract}

Background. In vitro experiments using radiolabeled molecules is fundamental for Positron emission tomography (PET) or single photon emission computed tomography (SPECT) tracer development and various metabolic assays, but no consensus on appropriate incubation conditions exists. Specifically, the use of shaking versus non-shaking conditions, cell number to medium volume and the choice of cell plating material may unintentionally influence cellular oxygenation and medium composition. This is problematic when testing the oxygen-dependence of tracers including 18F-fluoro-2-deoxyglucose (FDG) and hypoxia-selective 2-nitroimidazoles (e.g., 18F-fluoroazomycin-arabinoside, FAZA) or when doing prolonged experiments. The purpose of this study was to assess the influence of various experimental conditions on tracer retention. Methods. Tumor cells were seeded in a) Glass or standard Polystyrene Petri dishes or as b) discrete droplets in polystyrene Petri dishes or on $9 \mathrm{~mm}$ glass coverslips positioned in glass Petri dishes. When confluent, cells were pre-equilibrated for $2 \mathrm{~h}$ to $21 \%, 0.5 \%$ or $0 \% 02$ and FDG or FAZA was added, followed by cell harvest and analysis of radioactivity $1 \mathrm{~h}$ (FDG) or 3h (FAZA) after. Experiments were conducted with/without orbital shaking. Results. The influence of hypoxia on tracer retention varied widely among cell lines, but shaking-induced convection did not influence uptake. In contrast, hypoxia-driven FAZA, and to some extent FDG, retention was much lower in cells grown on polyethylene than glass. Scaling-down the number of cells did not compromise accuracy. Conclusions. Most experiments can be performed appropriately in the absence of shaking and with downscaling of cell number but the use of conventional plasticware is highly problematic for studies on tracers and drugs that are metabolized and retained or activated at low 02 levels.

\section{Background}

Numerous in vitro cellstudies involves assessment of radioactive tracers, but there is no consensus on how such experiments ideally should be performed, which in turn may depend on the application. In oncology, there is a special interest in the development of PET tracers that may allow us to quantify metabolic and microenvironmental differences between tumors. For example, the presence of viable hypoxic tumor cells in solid tumors is strongly linked to poor prognosis, and hypoxia driven PET tracer retention has been widely studied as a means to identify patients with hypoxic tumors [1]. Focus has mainly been on a) assessing the stimulation of anaerobic glycolysis in hypoxic cells which may principally be determined from FDG retention or $b$ ) the retention of hypoxia selective 2-nitroimidazoles which are reduced and retained at low oxygen levels [2]. In addition, FDG (or ${ }^{3} \mathrm{H}$ - or ${ }^{14} \mathrm{C}$-labeled glucose analogues) and other tracers have been used as a means to test cell metabolism and viability in a large number of drug development studies. However, the ideal in vitro incubation conditions for the testing of such tracers has not been defined and experiments have often been performed under conditions, where cellular hypoxia may be poorly controlled. For example, in dense cell cultures the actual cellular oxygen tension may be profoundly reduced compared to the oxygen tension in the equilibrating gas due to slow oxygen diffusion and high cellular respiration (so-called peri-cellular hypoxia). The confounding influence may be particular problematic when experiments are performed under low (but not zero) oxygen tensions. 
For example, mitochondrial respiration can be maintained down to very low oxygen levels with a half maximum respiration at $0.2-0-3 \mathrm{mmHg}$ in isolated mitochondria and $\sim 1 \mathrm{mmHg}$ in intact cells [3]. Thus, when doing experiments at low $\mathrm{O}_{2}$ levels, even minor differences between equilibration gasses and intracellular $\mathrm{O}_{2}$ caused by cellular respiration may profoundly influence energy metabolism. Diffusionlimitations may similarly affect the retention of tracers (and drugs in treatment experiments), which of course is of general importance and not only a concern for tracers that are tested for hypoxia-specificity. To what extent tracer diffusion-limitations are problematic, depends on tracer uptake capacity (e.g., slow transmembrane diffusion versus rapid active transport) and the size of the tracer, since large molecules will diffuse more slowly. Incubation under gentle orbital shaking conditions may effectively offset the problems with insufficient oxygen and tracer delivery, but the great majority of experiments are still conducted under non-shaking conditions. Low oxygen tensions protects against radio-induced DNA damage and during anoxic conditions radioresistance is typically 3-fold enhanced compared to welloxygenated conditions when experiments are performed in glass Petri dishes [4,5]. The radioprotective effect of anoxic incubation is much reduced in plastic Petri dishes and this finding has been attributed to the presence of significant amounts of oxygen in plastic (e.g., polystyrene) that may be released during incubation, especially for experiments with a rather short duration of the gas equilibrium period [5-7]. Nonetheless, several studies that have characterized the hypoxia-selectivity of tracers or hypoxiaactivated/hypoxia-selective cytotoxins (e.g., tirapazamine, $\mathrm{TH}-302$ ) have used plastic Petri dishes or well plates $[8,9]$. The primary purpose of this study was to assess and quantify the possible confounding influence of applying non-shaking conditions and traditional cell culturing plasticware.

\section{Materials And Methods}

The following cell lines were tested: SiHa (squamous cell carcinoma, SCC, of the cervix), FaDu $\mathrm{DD}_{\mathrm{DD}}$ (Head and Neck SCC), SW948 (colon adenocarcinoma, AC) and the mammary AC's MCF7 and MDAMB231. SiHa was cultured in MEM whereas remaining cell lines were cultured in HEPES-buffered DMEM. Medium was supplemented with $10 \%$ FCS, non-essential amino acids, pyruvate and penicillin/streptomycin. For experiments, cells were seeded in $5 \mathrm{~cm}$ Polystyrene or in Borosilicate glass dishes and grown until confluent (except SW948, which do not form dish-covering monolayers but rather tends to grow in clusters). For the testing of scaling-down the number of cells, while maintaining a high degree of confluence, cells were seeded as 5 discrete droplets of cells in $75 \mu$ medium in Polystyrene Petri dishes. Following attachment of cells $(>6 \mathrm{~h})$, dishes were flooded with medium and cells were grown until defined droplet areas were confluent. The chemical properties of Borosilicate glass Petri dishes makes seeding in discrete droplets challenging. Instead, $75 \mu$ cell suspension were seeded on $9 \mathrm{~mm}$ circular glass cover slips in compartmentalized (but with free fluid movement between compartments) glass Petri dishes with four compartments each holding one cover slip. Following attachment of cells ( $>6 \mathrm{~h})$, dishes were flooded with medium and cells were grown until confluent.

When ready for experiments, cells were supplied with $7.5 \mathrm{ml}$ pre-warmed fresh MEM (SiHa) or DMEM (other cell lines) medium and transported to the PET department and incubated without dish lids in gas 
tight chambers from Billups-Rothenberg, modified so that tracers can be added through small sealable holes while maintaining a defined gas atmosphere. Typically, experiments were done using gentle orbital shaking during the entire pre-equilibration and tracer retention periods. Following a $2 \mathrm{~h}$ pre-equilibration period, $20 \mu$ tracer, equivalent to $~ 0.5 \mathrm{MBq}$ for FDG and 1 MBq for FAZA, were added to each dish through the sealable holes using a Hamilton syringe. For $\mathrm{SiHa}$ and $\mathrm{FaDu}_{\mathrm{dd}}$, complementary experiments were also performed without shaking during the tracer retention period. In these experiments, cells were shaken for 30 min initially to ensure initial gas equilibration followed by a $90 \mathrm{~min}$ period without shaking. Immediately after tracer addition, cells where briefly shaken for 5 min to ensure appropriate mixing of tracer and medium. Cells were pre-incubated with $21 \% \mathrm{O}_{2}, 0.5 \% \mathrm{O}_{2}$ or $0 \% \mathrm{O}_{2}$ (all with $5 \% \mathrm{CO}_{2}$ and $\mathrm{N}_{2}$ as balance) delivered from gas flasks using a flow of $5 \mathrm{l} / \mathrm{min}$ for $30 \mathrm{~min}$ followed by $0.3 \mathrm{l} / \mathrm{min}$ for the duration of the experiment. To reduce evaporation, gas mixtures were humidified using glass gas washing bottles, which resulted in a total fluid loss by evaporation of less than $0.5 \%$ (unpublished observations, M Busk).

Following a tracer retention period of $1 \mathrm{~h}$ (FDG) or $3 \mathrm{~h}$ (FAZA), cells were harvested. In short, a $100 \mu \mathrm{l}$ medium sample, used for normalization, was drawn from each dish and transferred to scintillation vials. Afterwards, remaining medium was discarded and cells were rinsed thoroughly in saline by rapid submersion in three large beakers each containing $5 \mathrm{I}$ of isosmotic $\mathrm{NaCl}$. After wash, cells were collected in $0.75 \mathrm{ml}$ of water using cell scrapers and transferred to scintillation vials. Each dish was then carefully rinsed with an additional $0.75 \mathrm{ml}$ of water, which was transferred to their respective vials to ensure removal of any residual cell material. For coverslips, all cover slips from a given dish were washed and transferred to a single vial. Finally, radioactivity was determined using a Packard well counter.

\section{Calculations And Statistics}

All measurements were decay corrected and subsequently normalized to the activity concentration in the same dish to compensate for any unintentional dish-to-dish variability in tracer, which improves accuracy/reproducibility compared to assuming that activity concentration is identical in all dishes (unpublished observations, Busk M). Finally, cellular tracer retention was expressed relative to control cells $\left(21 \% \mathrm{O}_{2}\right)$. The influence of plating material (FAZA+FDG) and shaking conditions (FDG) were compared for a given cell line, plating approach (droplets/confluent dishes) and oxygenation level using a paired two-tailed T-test. The level of significance was $P<0.05$ and data are presented as means \pm standard error of mean (SEM).

\section{Results}

Total cellular tracer content at harvest was cell line dependent but was consistently below $2 \%$ for FAZA and $1 \%$ for FDG in confluent Petri dishes suggesting that tracer availability is buffered sufficiently for the entire labeling period. Scaling down cell number, by reducing the effective growth area, further reduced retention by 3-6 fold. Figure 1 shows the oxygen dependence of cellular FAZA uptake in confluent cells, expressed as ratios between hypoxic and well-oxygenated cells following a $3 \mathrm{~h}$ tracer-loading period. Low 
oxygen levels stimulated FAZA retention profoundly, but with distinct intra-cell line differences. Of note, uptake was much lower in cells grown on polystyrene than on glass, and stimulation under $0.5 \% \mathrm{O}_{2}$ on glass was similar or even higher than uptake in cells in polystyrene dishes equilibrated to an anoxic gas. We also assessed the influence of plating material on the oxygenation-dependent stimulation of glycolysis (from FDG retention) in a cell line panel (figure 2 and 3), and for FaDu $\mathrm{DD}_{\text {and }} \mathrm{SiHa}$ cells we extended the experimental design to also include a comparison between stagnant and shaking conditions, as well as the reliability of downscaling of cell number (figure 2). The hypoxia-induced stimulation of FDG retention varied widely between cell lines, ranging from a barely detectable response in MDAMB-231 (figure 3) to a 10 fold increase under anoxia in SiHa cells (figure 2) grown on glass. The influence of plating material was less pronounced for FDG than for FAZA, but polystyrene suppressed the stimulatory effect of hypoxia in SiHa cells significantly. Reducing the cell-number-to-medium volume, while maintaining confluence, by downscaling of the effective growth area, was robust and did not affect hypoxic-to-normoxic tissue ratios nor inter-experiment variability (figure 2).

\section{Discussion}

Our results showed that plating material influence the hypoxia-driven retention of FAZA profoundly in all cell lines, whereas a suppressive effect of polystyrene on FDG uptake only was observed in SiHa cells. Of note, FAZA retention data suggests that the average effective cellular oxygen pressure experienced during the tracer loading period is above $0.5 \% \mathrm{O}_{2}$ in polystyrene attached cells even after a $2 \mathrm{~h}$ pre-equilibration period under shaking conditions. This equals a $\mathrm{pO}_{2}$ of $\sim 3.5 \mathrm{mmHg}$ assuming a normal pressure of 760 $\mathrm{mmHg}$ and a water vapor pressure of $47 \mathrm{mmHg}$ when using a gas humidifier. Such differences may influence any experiment where exact control of oxygen levels are critical, including studies on hypoxiainduced gene expression and hypoxia activated prodrugs. Our results do not decisively rule out other explanations for reduced anoxia-driven tracer uptake in polystyrene grown cells, but our observations corroborate with seminal studies showing that radiosensitivity during an anoxic gas challenge is elevated when using polystyrene and that it relates to the presence of substantial and releasable quantities of $\mathrm{O}_{2}$ in plastics [6,7]. Other types of plastic such as permanox may reduce, but not fully eliminate, the problem [10]. To avoid unwanted influx of oxygen from the substrate, pre-equilibrated plastic-ware has been applied in some studies on bioreductive cytotoxins and the kinetics of hypoxia-induced genes [11]. However, such an approach requires a hypoxia work-station and more importantly, prevents seeding and growing of cells under identical standard culturing conditions prior to experimentation and gas challenge, which in turn may induce unknown biological differences and experimental bias that cannot be resolved unequivocally. The confounding influence of plastic-ware in hypoxia research have generally gained footing in radiotherapy research, but is less appreciated outside this field. In most cell lines, FDG uptake was uninfluenced by plating material, suggesting that glycolysis is fully activated at $\mathrm{pO}_{2}$ levels higher than those required for maximal reduction of FAZA. This is somewhat surprising since the partial pressure at which respiration rate is $50 \%$ of maximum is reported to be below $1 \mathrm{mmHg}$ in intact cells [3], but stimulation of glycolytic flux may proceed cessation of mitochondrial energy production. Interestingly, in $\mathrm{SiHa}$ cells, which unlike typical tumor cells have a largely non-glycolytic phenotype under aerobic 
conditions, and thus experience larger adaptive glycolytic flux changes when mitochondrial ATP synthesis ceases, a significant difference was observed between polystyrene and glass. Whether this relates to true cell-to-cell line differences in $\mathrm{pO}_{2}$ threshold values that affects respiration or rather reflects increased assay sensitivity in the highly responsive SiHa cell line is unclear, but highlights that also in studies on hypoxia-induced changes in energy metabolism culturing material is of importance. Indeed, in such studies the use of well-plates is common and this may further exaggerate the problem of prolonged oxygen release and also result in differences between centrally and peripherally located wells, so-called edge effects. Of note, in a hypothetical study using only polystyrene as substrate, one would erroneously conclude that FDG and FAZA performs equally well as markers of severe hypoxia in SiHa cells (compare figure 1 and 2) and that FAZA displays no hypoxia-selectivity in MDAMB-231 cells (figure 1).

Another, often underappreciated, problem is that cells alter their local and global environment by consumption of tracers, $\mathrm{O}_{2}$, metabolites and possibly even drugs, which may lead to insufficient diffusive delivery of these substances. During long-term experiments in dense cultures alterations in medium composition (e.g., glucose) may also be a concern (see also next paragraph). For example, Pettersen et al. [12] showed that pericellular oxygen tension in confluent stagnant cultures may deviate substantially from $\mathrm{pO}_{2}$ in equilibration gasses, and concluded that this may affect any study that correlates cell biology to oxygen levels. One approach to optimize control of the pericellular microenvironment, is the use of freshly trypsinized cells maintained in shaken suspension in glass vials. This procedure has been valuable in several settings including the testing of hypoxia PET tracers $[13,14]$, but may be less useful for delicate metabolic and gene expression studies, since several studies have shown that detached cells are stressed, and may behave differently than attached cells $[15,16]$. Therefore, when possible the use of attached cells are ideal. Not all cells attach firmly to glass, but coating may effectively overcome these problems. A simple, but not commonly used, way to overcome or limit diffusion barriers is the use of gentle orbital shaking, since convection will ensure fast and effective gas equilibration of medium and reduce the effective thickness of still layers. This may be particularly relevant when studying low, but nonzero, levels of oxygenation, where the relative influence by cell self-consumption may lead to large relative changes in oxygenation. Surprisingly, our results showed that FDG retention was unaffected by shaking conditions even at $\mathrm{O}_{2}$ of $0.5 \%$ in dense cultures. A similar result was obtained for FAZA retention in $\mathrm{SiHa}$ cells ( $n=2$; unpublished observations, Busk M). These results suggests that even in experiments where proper control of $\mathrm{pO}_{2}$ is required non-shaking conditions may be appropriate.

A key advantage of using tracers such as FDG, ${ }^{11} \mathrm{C}$-acetate and ${ }^{11} \mathrm{C}$-methionine to assess cellular metabolism, is that measurements can be performed rapidly under reasonably stable culturing conditions. In contrast, traditional enzymatic assays determines uptake rates based on concentration changes in metabolites in medium samples, which by necessity prevents measurements of fluxes at stable medium and cell number conditions. A second problem is that detectable changes in medium composition may require long incubation periods, which leads to substantial evaporation even when using humidified gasses. In our acute experiments, the total cellular tracer retention was typically below $2 \%$ of total added dose (quantified in medium samples prior to cell harvest), suggesting that medium- 
volume-to-cell content was sufficient to ensure near stable conditions. Nonetheless, in many settings, such as studies on cell metabolism under chronic tumor-microenvironment-mimicking conditions with nutritional deprivation, further reducing the cell number may be advantageous or even required to dampen inappropriate changes in medium metabolites that affects tracer retention by competition (e.g., glucose, acetate) or indirectly by changing cellular metabolic state (e.g., lactic acidosis). In accordance, we also assessed the reliability of downscaling the number of cells, while maintaining a high degree of confluence, by growing cells in discrete areas in polystyrene dishes or on glass cover slips. Our results shows that such downscaling was reliable resulting in similar results as obtained when growing cells as dense cultures in whole dishes. An added advantage is that cell washing and radioactivity assessment is very easy to perform when using cover slips since cell scraping and collection is not required, and that cells do not, or only slowly, spread to the Petri dish bottom. The coverslip approach is thus particular useful when doing prolonged incubations where strictly defined environmental conditions are required at a high degree of confluence. In cells on cover slips, SiHa typically retained around $0.25 \%$ of added FDG under anoxia in $1 \mathrm{~h}$ in MEM $(\approx 5 \mathrm{mM}$ glucose). Assuming that the relative affinity for FDG retention and glucose use (the so-called "lumped constant") is one [17] and that glucose metabolic rate is maintained under hypoglycemic conditions, then a $24 \mathrm{~h}$ anoxic pre-incubation of SiHa cells at $1 \mathrm{mM}$ glucose would lower medium glucose by approximately $30 \% \mathrm{mM}$. This quantity can be further reduced to $\sim 7.5 \%$, when only using a single cover slip, suggesting that by appropriate downscaling such experiments are feasible.

\section{Conclusions}

We have demonstrated that choosing an appropriate plating material is crucial for obtaining reliable results in studies involving hypoxia, whereas orbital shaking may be omitted. Robustness was not compromised by down scaling, which may be required in prolonged studies where careful control of the medium composition is required.

\section{Declarations}

\section{Abbreviations}

FDG: ${ }^{18}$ F-fluoro-2-deoxyglucose

FAZA: ${ }^{18}$ F-fluoroazomycin-arabinoside

PET: positron emission tomography

SPECT: single photon emission computed tomography

SCC: squamous cell carcinoma

AC: adenocarcinoma 
MEM: Minimum Essential Medium Eagle

DMEM: Dulbecco's Modified Eagle's Medium

HEPES: 4-(2-Hydroxyethyl)piperazine-1-ethanesulfonic acid

\section{Ethics approval and consent to participate}

Not applicable

\section{Consent for publication}

Not applicable

\section{Availability of data and material}

All data generated or analysed during this study are included in this published article.

\section{Competing interests}

The authors declare that they have no competing interests

\section{Funding}

This study was supported by an unrestricted grant from the Danish Cancer Society [Grant number: R90A6196]

\section{Acknowledgements}

Not applicable

\section{Authors' contribution}

M Busk designed the study, conducted the experiments, performed the analyses and drafted the manuscript. S Jakobsen synthetized the tracers. All authors contributed to manuscript revision and approved the final manuscript. 


\section{References}

1.Horsman, M. R., Mortensen, L. S., Petersen, J. B., Busk, M. \& Overgaard, J. Imaging hypoxia to improve radiotherapy outcome. Nat. Rev. Clin. Oncol. 9, 674-687 (2012).

2.Busk, M. et al. Cellular uptake of PET tracers of glucose metabolism and hypoxia and their linkage. Eur. J. Nucl. Med. Mol. Imaging 35, 2294-2303 (2008).

3.Steinlechner-Maran, R., Eberl, T., Kunc, M., Margreiter, R. \& Gnaiger, E. Oxygen dependence of respiration in coupled and uncoupled endothelial cells. Am. J. Physiol. 271, C2053-61 (1996).

4.Sorensen, B. S. et al. Radiosensitivity and effect of hypoxia in HPV positive head and neck cancer cells. Radiother. Oncol. 108, 500-505 (2013).

5.Gray, L. H., Conger, A.D., Ebert, M., Hornsey, S. \& Scott, O. C. The concentration of oxygen dissolved in tissues at the time of irradiation as a factor in radiotherapy. Br. J. Radiol. 26, 638-648 (1953).

6.Chapman, J. D., Sturrock, J., Boag, J. W. \& Crookall, J. O. Factors affecting the oxygen tension around cells growing in plastic Petri dishes. Int. J. Radiat. Biol. Relat. Stud. Phys. Chem. Med. 17, 305-328 (1970).

7.Chapman, J. D., Sturrock, J., Boag, J. W. \& Crookall, J. O. The oxygen tension around mammalian cells growing on plastic Petri dishes and its effect on cell survival curves. Br. J. Radiol. 42, 399 (1969).

8.Kumar, S. et al. Hypoxia-Targeting Drug Evofosfamide (TH-302) Enhances Sunitinib Activity in Neuroblastoma Xenograft Models. Transl. Oncol. 11, 911-919 (2018).

9.Sun, J. D. et al. Combination treatment with hypoxia-activated prodrug evofosfamide (TH-302) and mTOR inhibitors results in enhanced antitumor efficacy in preclinical renal cell carcinoma models. Am. J. Cancer Res. 5, 2139-2155 (2015).

10.Zeman, E. M., Pearson, C. I. \& Brown, J. M. Induction of hypoxia in glass versus Permanox petri dishes. Radiat. Res. 122, 72-76 (1990).

11.Jamieson, S. M. et al. Evofosfamide for the treatment of human papillomavirus-negative head and neck squamous cell carcinoma. JCl insight 3, (2018).

12.Pettersen, E. O., Larsen, L. H., Ramsing, N. B. \& Ebbesen, P. Pericellular oxygen depletion during ordinary tissue culturing, measured with oxygen microsensors. Cell Prolif. 38, 257-267 (2005).

13.Minn, H., Clavo, A. C., Fisher, S. J. \& Wahl, R. L. Effect of nitroimidazole sensitizers on in vitro glycolytic metabolism of hypoxic squamous cell carcinoma. Acta Oncol. 39, 199-205 (2000).

14.Minn, H., Clavo, A. C. \& Wahl, R. L. Influence of hypoxia on tracer accumulation in squamous-cell carcinoma: in vitro evaluation for PET imaging. Nucl. Med. Biol. 23, 941-946 (1996). 
15.Danhier, P. et al. Influence of cell detachment on the respiration rate of tumor and endothelial cells. PLoS One 8, e53324 (2013).

16.Ren, X.-D. et al. Disruption of Rho signal transduction upon cell detachment. J. Cell Sci. 117, 35113518 (2004).

17.Barrio, J. R. et al. Does 2-FDG-PET Accurately Reflect Quantitative In vivo Glucose Utilization? J. Nucl. Med. (2019) doi:10.2967/jnumed.119.237446.

\section{Figures}



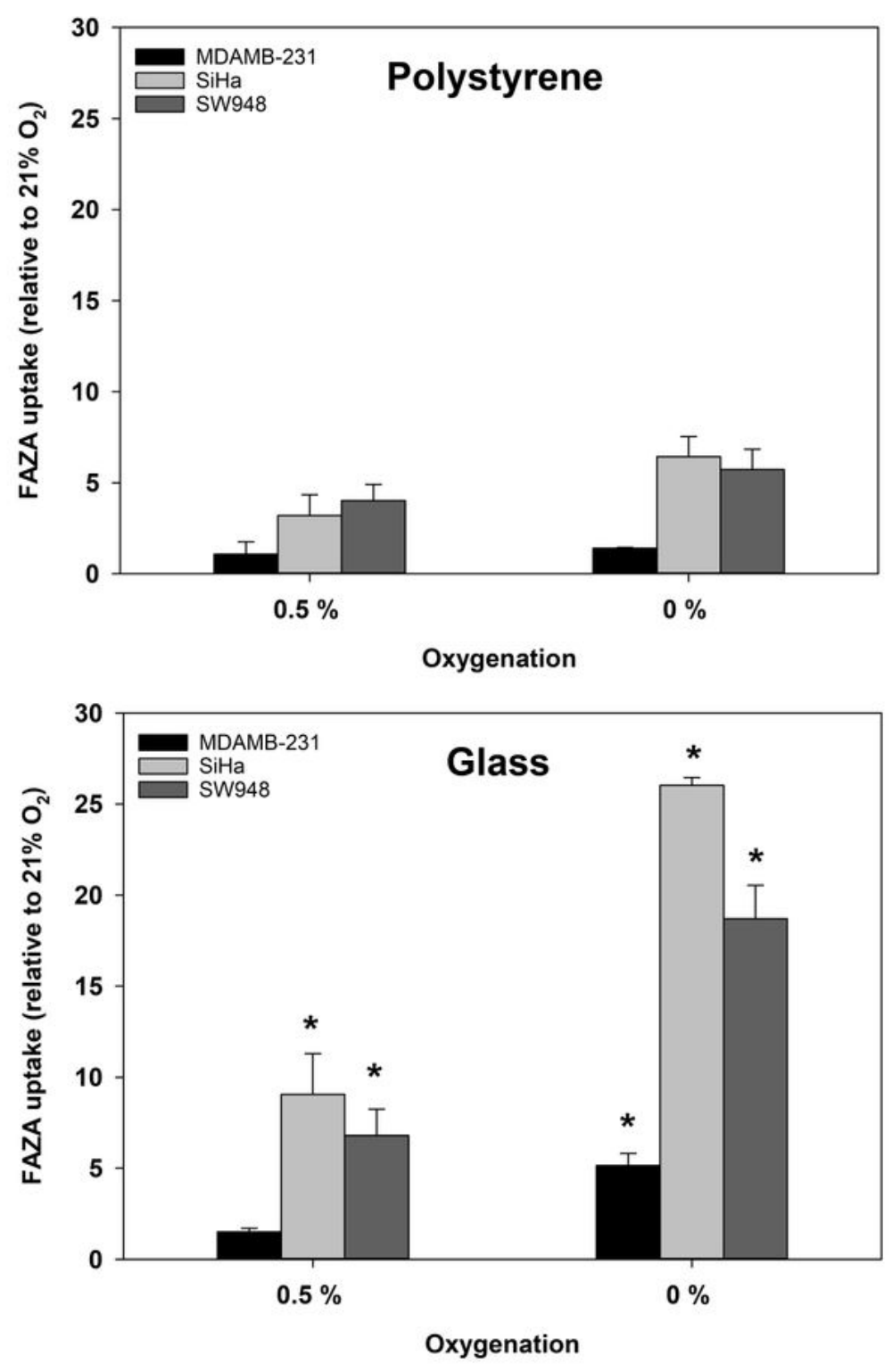

\section{Figure 1}

Oxygenation-dependent FAZA binding and its dependency on plating material. FAZA uptake under hypoxic $(0.5 \% 02)$ and anoxic $(0 \% 02)$ gas equilibration is expressed relative to the radioactivity measured in well-oxygenated $(21 \% 02)$ cells. Values are means $( \pm$ SEM) of three to four independent experiments. The asterisks denotes a significant difference between glass and polystyrene at a given oxygenation level $(p<0.05)$. 

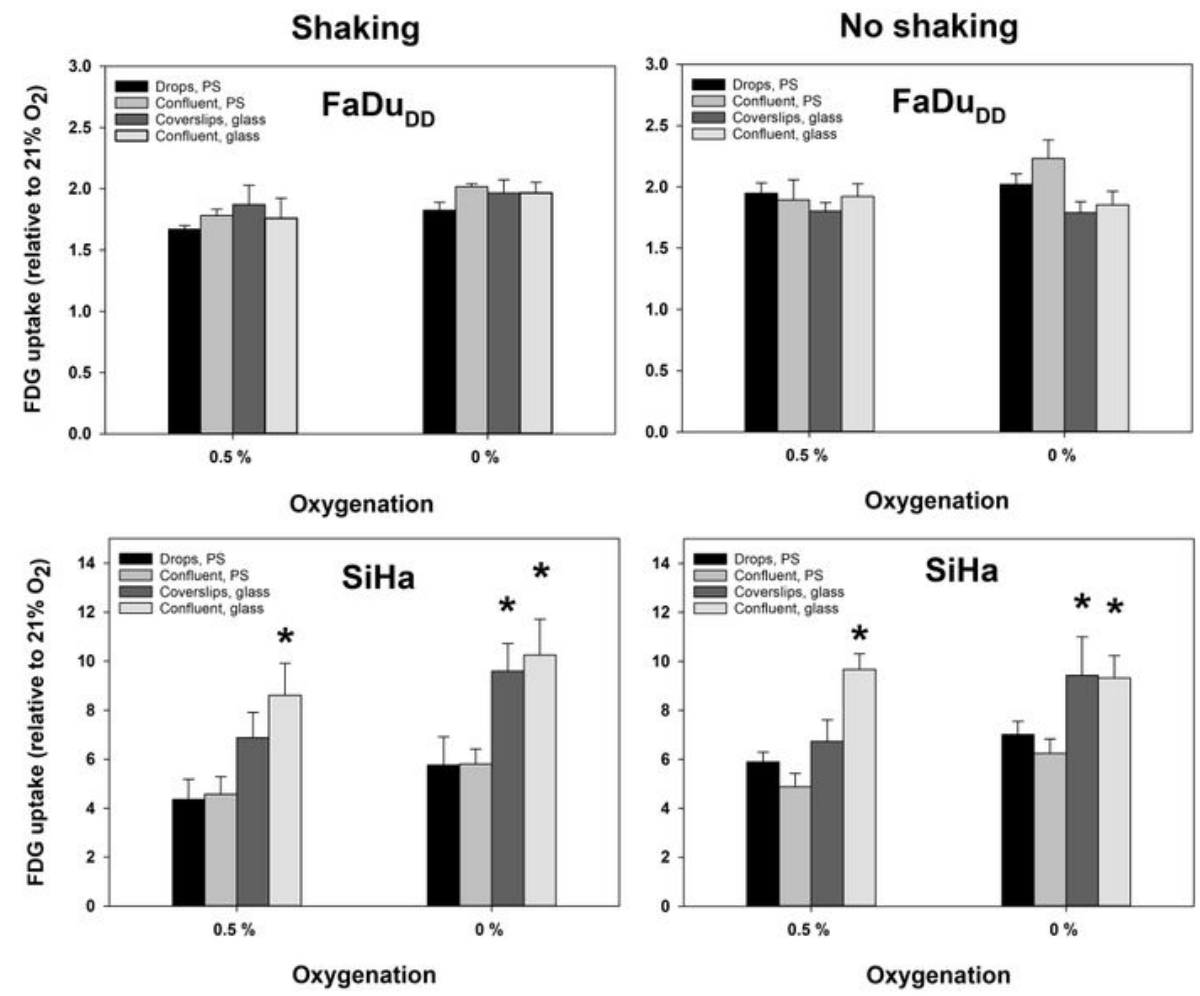

\section{Figure 4}

Oxygenation-dependent FDG uptake and its dependency on plating material, shaking conditions and cell number (growth area). FDG retention under hypoxic $(0.5 \% 02)$ and anoxic $(0 \% 02)$ gas equilibration is expressed relative to the radioactivity measured in well-oxygenated $(21 \% 02)$ cells. Values are means $( \pm$ SEM) of three to four independent experiments. The asterisks denotes a significant difference between 
glass and polystyrene at a given oxygenation, cell number (growth area) and shanking condition ( $\mathrm{p}<$ 0.05).
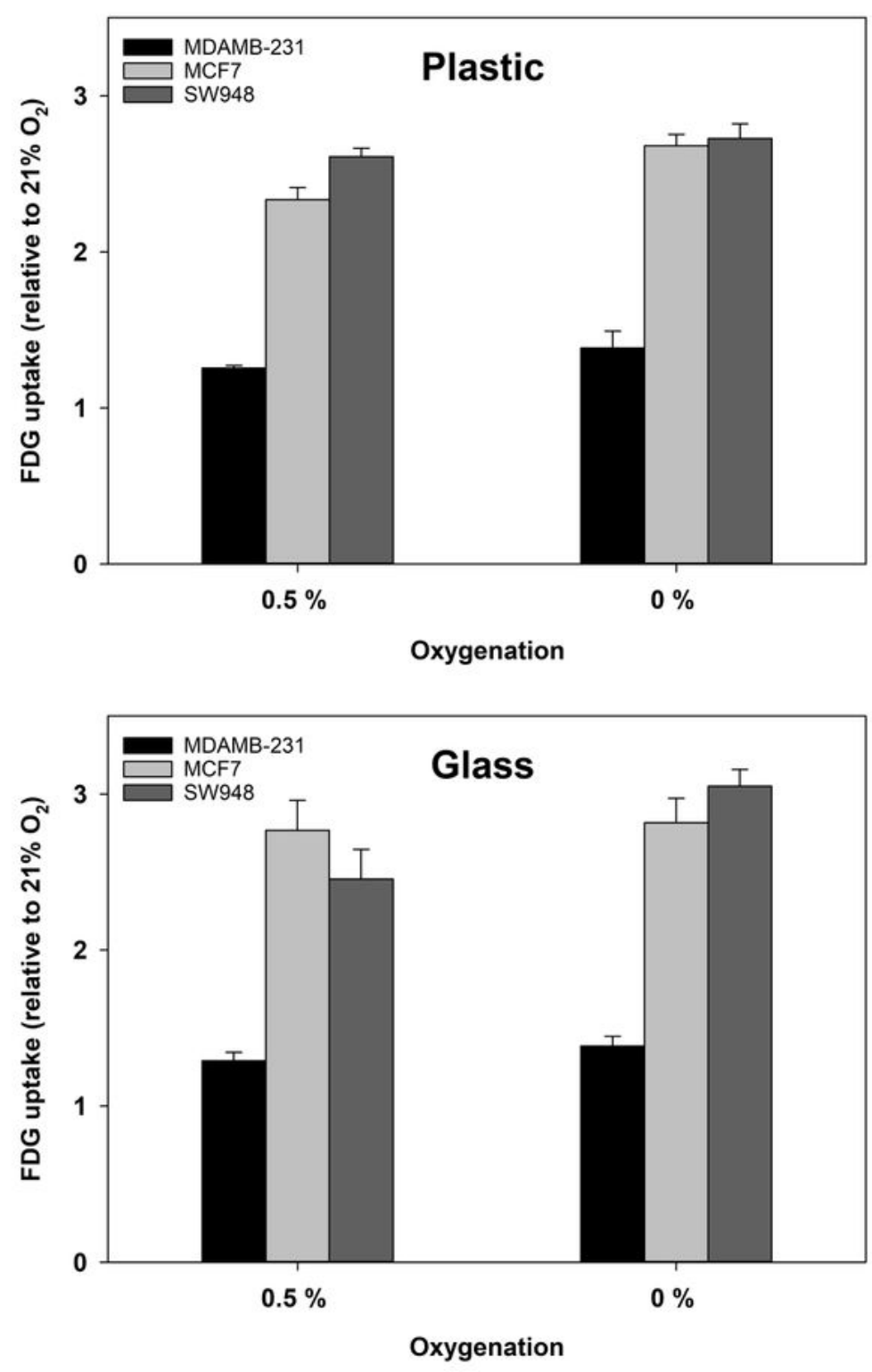

Figure 6

Oxygenation-dependent FDG uptake and its dependency on plating material using orbital shaking in a further panel of cell lines. Values are means $( \pm$ SEM) of three to four independent experiments. 